\title{
Articles
}

\section{Loss of early insulin secretion leads to postprandial hyperglycaemia}

\author{
S. Del Prato \\ Department of Endocrinology and Metabolism, Section of Diabetes, Ospedale Cisanello, Pisa, Italy \\ Department of Endocrinology and Metabolism, Section of Diabetes, School of Medicine, University of Pisa, Italy
}

\section{Abstract}

Aims/Hypothesis. A loss of early-phase insulin response is common in Type 2 diabetic patients and in people with impaired glucose metabolism. It is hypothesized that this alteration is not simply a marker for the risk of developing diabetes, rather it is a an important pathogenetic mechanism causing excessive postprandial hyperglycaemia.

Methods. Relevant literature on the epidemiology, physiopathology, and therapeutic intervention related to loss of early insulin secretion and postprandial hyperglycaemia has been analysed.

Results. In response to intravenous glucose, insulin secretion is biphasic. This behaviour translates as a rapid release of insulin into the blood stream in response to the ingestion of carbohydrates or a mixed meal. The rapid increase in portal insulin concentration and the avid binding of the hormone to its receptor on liver cell membranes, account for a prompt suppression of endogenous glucose production and reduction of the rate of increase in plasma glucose concentrations. This has been supported by experimental studies carried out in both animals and humans: the selective abolition of early insulin secretion in healthy subjects results in impaired glucose tolerance, excessive glucose excursions, and possible hampering of the thermic effects of ingested carbohydrates. In nondiabetic subjects, the loss of early insulin secretion is a determinant for developing diabetes. The critical role of the early-phase insulin response in determining postprandial hyperglycaemia, is supported by an amelioration of glucose tolerance by restoring the acute rise in plasma insulin concentrations after the ingestion of both glucose and a mixed meal. This amelioration in plasma glucose profile can prevent late hyperglycaemia and hyperinsulinaemia.

Conclusion/interpretation. Therapeutic approaches aimed at restoring a physiological pattern of insulin secretion could prove effective in reducing postprandial glucose excursions particularly in the early stage of Type 2 diabetes. [Diabetologia (2003) 46[Suppl1]:M2-M8]

Keywords Type 2 diabetes, early-phase insulin secretion, plasma glucose, glucose excursions, postprandial hyperglycaemia, glucose tolerance, plasma insulin, hyperinsulinaemia.
Received: 17 April 2001 / Revised: 24 September 2001 Published online: 12 November 2002

CC Springer-Verlag 2002

Corresponding author: S. Del Prato, Department of Endocrinology and Metabolism, Section of Diabetes, Ospedale Cisanello, Via Paradisa, 2, 56124 Pisa, Italy, E-mail: delprato @immr.med.unipi.it

Abbreviations: EGP, Endogenous glucose production; IVGTT, intravenous glucose tolerance test; FPG, fasting plasma glucose; AIR, acute insulin response.
Type 2 diabetes mellitus is a heterogeneous condition characterised by a dual pathogenetic mechanism: insulin resistance and defective insulin secretion. Insulin resistance is believed to precede the development of diabetes. Low insulin sensitivity has been found in normoglycaemic relatives of Type 2 diabetic patients $[1,2]$ and to predict the development of clinically overt hyperglycaemia [3]. Loss of compensatory hypersecretion of insulin has been indicated as the turning point for the development of diabetes [4]. However, alterations in insulin secretion have been 


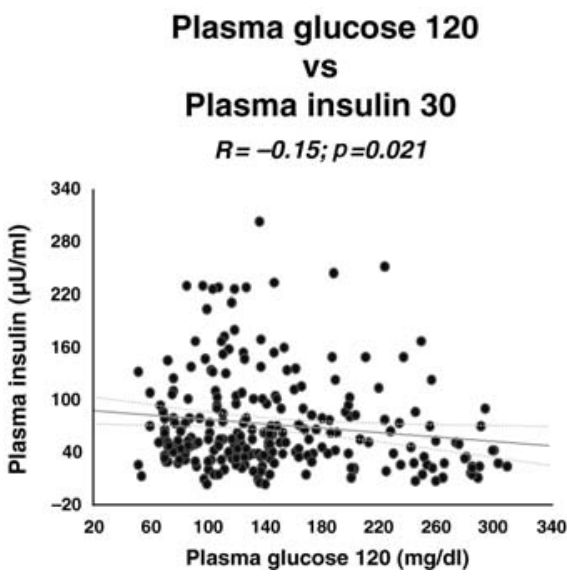

Fig. 1. Correlation of 30-min plasma insulin and C-peptide response with 2-h plasma glucose concentrations after an OGTT in a population including subjects with normal and impaired glucose tolerance and diabetes

described that precede diabetic hyperglycaemia. In particular, the loss of the acute phase of insulin secretion is found in the very early stages of the disease. This paper is a review of literature on the physiology of insulin secretion, metabolic implications of the loss of early-phase insulin secretion and its role in the hyperglycaemia of Type 2 diabetic patients.

\section{Physiology of insulin secretion}

In response to a glucose challenge, both in vivo and in vitro, insulin is rapidly secreted reaching maximal secretion rate within a few minutes after glucose exposure [5]. The secretory response is greater after oral than parenteral glucose load. This effect is due to the activation of the entero-insular axis and the amplifying effect of gut incretins on insulin release [6]. The strict time-dependency between insulin secretion and glucose challenge becomes even more striking if the cephalic phase of the hormone release is taken into account. In healthy people, insulin increase precedes the nutrient ingestion due to the activation of neuronal and neuro-endocrine stimuli. Though modest in absolute terms, this rise in plasma insulin concentration seems to play an important physiological role, as an inverse relation between the cephalic phase of insulin secretion and the initial rise in plasma glucose concentration has been reported [7]. Following the ingestion of nutrients, insulin secretion becomes quantitatively relevant.

In response to an i.v. glucose challenge, insulin secretion is characteristically biphasic [5]. Within minutes of ingestion of a glucose load, insulin is rapidly secreted reaching an initial peak value in 5 to $7 \mathrm{~min}$. This early phase of insulin release lasts no more than 10 to $15 \mathrm{~min}$ and is followed by a more sustained secretion lasting several hours, until the stimulus is
Plasma glucose 120

vs

Plasma C-peptide 30

$R=-0.22 ; p=0.001$

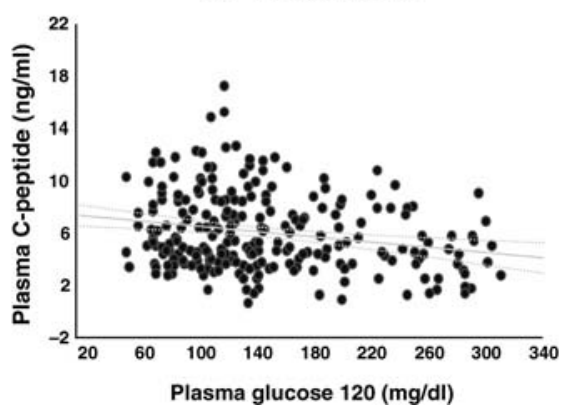

removed or the plasma glucose concentration has returned to baseline values. Approximately 2 to $3 \%$ of beta-cell insulin content is released during the earlyphase secretion, while the second phase accounts for approximately $20 \%$ of the beta-cell content.

\section{Early-phase insulin secretion and glucose homeostasis}

Early-phase insulin secretion plays a critical role in the maintenance of glucose homeostasis. A strong negative relation has been found between cephalic insulin release and initial glucose increment after a glucose infusion [7]. This finding suggested that an early surge of insulin secretion, such as the cephalic phase, could exert a restraining effect on rising blood glucose concentrations. Further, the greater the insulin surge, the more prolonged the effect on glucose homeostasis. After the ingestion of an oral glucose load, an inverse relation was noted between 30 -min plasma insulin concentration (a marker for early insulin secretion) and 2-h plasma glucose concentrations [8]. Conversely, a direct relation was noted between the plasma insulin and glucose concentration in the second hour. These observations indicate that early rapid insulin rise is critical in restraining excessive glucose excursions after nutrients are ingested. In the presence of a weak early-phase insulin secretion, plasma glucose will progressively rise causing sustained insulin release needed for the plasma glucose concentration to return towards baseline. These results have been replicated in larger populations. In a group of 230 people with various degrees of glucose tolerance (Fig. 1), 30-min post-OGTT plasma insulin or C-peptide concentration was inversely related to the 2 -h plasma glucose concentration $(p<0.05)$ [9]. In conclusion, defective early rise in plasma insulin concentrations could lead to hyperglycaemia and hyperinsulinaemia.

The dimension and time-course of insulin release during early-phase secretion is compatible with an effect on the liver rather than on peripheral tissues. Animal studies support this view [10]. In those experiments, plasma glucose concentration and endogenous 
glucose production (EGP) were measured in glucagon-infused dogs undergoing a pancreatic islet clamp, in which insulin secretion was completely abolished or either the first, second, or both phases of insulin secretion were reconstructed. The study results showed that both first-phase and second-phase insulin secretion were critical in counterbalancing the hyperglycaemic effect of glucagon, but isolated restoration of the early rise in plasma insulin concentration could reduce the glucagon-mediated rise in plasma glucose concentration. In a subsequent study, the same authors showed that the primary effect on EGP was the suppression of gluconeogenesis [11], an important finding considering the characteristic increase in gluconeogenesis of Type 2 diabetes [12].

Human studies also lend support to the central role of early-phase insulin secretion in the modulation of EGP. Hyperglycaemic clamp studies have been carried out in normal subjects together with radioactive glucose tracer infusion for measurements of glucose production and uptake [13]. The acute increase of plasma glucose concentration elicited the characteristic biphasic insulin release causing progressive EGP suppression and increase in glucose disposal through peripheral tissues. The abolition of the early-phase insulin secretion by means of somatostatin and appropriate insulin replacement did not affect glucose utilisation. On the contrary, glucose appearance in the circulation proceeded at higher rates in spite of the prevalent hyperglycaemia and hyperinsulinaemia: suppression of EGP was only $50 \%$ as compared with the control study (Fig. 2). Impaired EGP suppression was a direct consequence of the deficit of early-phase insulin secretion, as proven by restoration of early insulin rise in plasma.

Though the biphasic response is fully appreciated with an i.v. glucose challenge, a rapid increase in plasma insulin concentrations after an oral glucose load seems to be essential for normal glucose tolerance. An experimental approach similar to [13] was used in other studies [14], with the exception that an OGTT or an intravenous glucose tolerance test (IVGTT) were carried out. Early insulin release was completely blocked by somatostatin infusion in both cases. The loss of the early phase of insulin secretion was associated with a deterioration of glucose tolerance as indicated by the $\mathrm{K}$ value $(1.9 \pm 0.4$ vs $1.1 \pm 0.3, p<0.001)$. Moreover, in response to the OGTT a larger glycaemic excursion occurred. Surprisingly, glucose-induced thermogenesis was reduced in association with the loss of the early phase of insulin release. The increase in energy expenditure could suggest that restoration of early phase insulin secretion is of importance in activating the membrane bound $\mathrm{Na}^{+}-\mathrm{K}^{+}$-ATPase and/or by stimulating the sympathetic nervous system. By using a more sophisticated technique, similar results were found [15]. Non-diabetic subjects were studied during somatostatin inhibition of endogenous insulin secre-
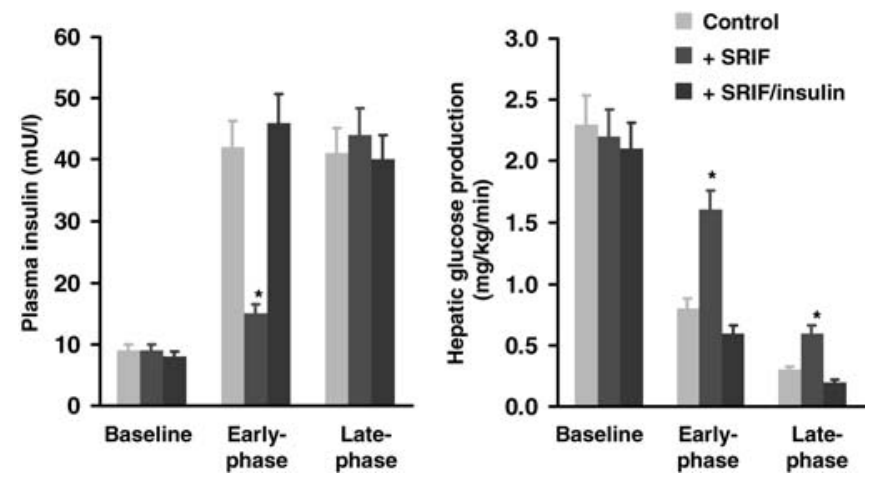

Fig. 2. Means ( \pm SE) plasma insulin and HPG in healthy subjects during control hyperglycaemic clamp; hyperglycaemic clamp plus somatostatin and insulin infusion mimicking only second-phase insulin secretion, and hyperglycaemic clamp plus somatostatin and insulin infusion mimicking both firstphase and second-phase insulin secretion; Adapted from Luzi L, et al. [13]

tion, while exogenous insulin was infused so as to mimic postprandial insulin profiles of a typical healthy or diabetic subject. Simultaneously, glucose was infused in a pattern and amount that mimicked the systemic delivery rate normally observed after the ingestion of $50 \mathrm{~g}$ of glucose. The delayed pattern of insulin delivery (i.e. loss of early-phase insulin secretion) was associated with higher $(p<0.05)$ glucose concentration and prolonged duration of hyperglycaemia. This alteration was mainly due to altered suppression of EGP.

Furthermore, after the ingestion of a mixed meal, plasma insulin concentration increases rapidly with a 30-min peak [16]. The quick rise in plasma insulin concentration is associated with a marked decrease in the glucagon to insulin ratio and concomitant suppression of EGP and glycogen accretion in the liver.

In summary, both animal and human studies support the critical physiological role of the early phase of insulin secretion in the maintenance of postmeal glucose homeostasis. This effect is primarily mediated at the level of the liver, allowing prompt inhibition of EGP and thereby restraining the mealtime plasma glucose excursion.

\section{Early loss of early-phase insulin secretion in Type 2 diabetes mellitus}

Type 2 diabetes is a heterogeneous disorder characterised by defects in beta-cell function and insulin action. Insulin resistance is a key feature of Type 2 diabetes as it can be in the vast majority of affected people [17]. Impaired insulin action occurs at the level of all insulin-dependent tissues: liver, muscle and adipose tissue. These abnormalities are likely the consequence of molecular defects at the level of postreceptor signalling, glucose transport, and/or regulatory enzymes of intracellular glucose metabolism [18]. 
Insulin resistance by itself does not seem to be sufficient to cause diabetes. Longitudinal studies have shown that the development of overt hyperglycaemia is also associated with a decline in beta-cell secretion [17, 19]. Patients who have Type 2 diabetes with a fasting plasma glucose (FGP) of less than $7.8 \mathrm{mmol} / \mathrm{l}$ can have a similar or even greater insulin response to glucose than matched healthy subjects; however, the overall plasma insulin concentrations are always inadequate compared with prevalent plasma glucose concentration [17]. An absolute defect in insulin secretion becomes apparent at FPG concentrations of more than $10 \mathrm{mmol} / \mathrm{l}$. When hyperglycaemia develops, it exerts a toxic effect on both the pancreas and insulin-sensitive tissues, a phenomenon known as 'glucose toxicity' [20], leading to a self-perpetuating cycle of worsening defects and further exacerbation of the hyperglycaemic condition.

In patients with IGT or in the early stages of Type 2 diabetes, early-phase insulin release is almost invariably lost despite the enhancement of the secondphase secretion $[21,22]$. The results of the insulin response to glucose tolerance tests from 32 studies in patients with Type 2 diabetes mellitus has been summarized [17]. In terms of the total insulin response, insulin secretion was reduced in 16 of the studies, normal in 11, and increased in only 5 of them. A different picture emerged when early-phase (0-10 min) versus late-phase insulin secretion was examined. Late-phase insulin secretion was decreased in 13 studies, whereas normal or even increased responses were reported in the remaining 19 studies. In contrast, the majority of the reports $(n=21)$ showed a defective early phase of insulin secretion.

The loss of rapid insulin release is a determinant of the development of Type 2 diabetes. Among a cohort of 348 women aged 50 on entering a 12-year prospective study, the incidence of diabetes was higher in those who initially had higher FPG and insulin concentrations and lower early insulin release in response to IVGTT [23]. In particular, $17.1 \%$ of the women who had early insulin response below the lowest quintile developed diabetes.

The insulinogenic index is calculated as the ratio of the increment of plasma insulin to glucose concentration 30 min after an OGTT, and it provides a parameter of early insulin response which correlates with the acute insulin response to IVGTT [24]. Low values of the insulinogenic index precede the increase of FPG concentrations [24]. These results are similar to our observations. By employing a minimal model analysis, a parameter of beta-cell response ( $\beta$-index) was derived from plasma glucose and $\mathrm{C}$-peptide concentration during an OGTT [25] in a large cohort of subjects with various degrees of glucose tolerance. The $\beta$-index progressively declined from the condition of NGT to IGT, to overt diabetes [26]. Moreover, the $\beta$-index was lower in subjects with a family history of
Type 2 diabetes than those without a family history, suggesting that genetic factors are important determinants of the insulin secretory response.

More recently, longitudinal data from 17 Pima Indians in whom glucose tolerance deteriorated from NGT to IGT to diabetic over a 5-year follow-up have been reported [27]. The transition from NGT to IGT was associated with an increase in body weight, a decline in insulin-stimulated glucose disposal and a reduction of acute insulin response (AIR) to intravenous glucose. Progression from IGT to diabetes was associated with a modest worsening in insulin sensitivity but a much greater decline in AIR. Thirty-one subjects who retained NGT over a similar period also gained weight and their insulin sensitivity had deteriorated further, but their AIR increased.

This brief synopsis illustrates the altered insulin secretion dynamics in Type 2 diabetes, underscoring the deficient acute early phase of insulin release.

\section{Restoration of early insulin release in Type 2 diabetic patients}

If the loss of early insulin release plays a major role in the pathogenesis of postprandial hyperglycaemia, therapeutic intervention to restore it should be able to improve glucose tolerance in Type 2 diabetic patients.

A study [28] was designed to test whether correcting the deficiency in early prandial insulin secretion with a physiological amount of exogenous insulin would have a beneficial effect on postprandial glucose excursions in Type 2 diabetic patients. For this purpose, the same amount of insulin was given intravenously at mealtime to diabetic patients: (i.) over $30 \mathrm{~min}$ at the beginning of a meal (i.e. restoration of early insulin increase), (ii.) with the same insulin profile but delayed by $30 \mathrm{~min}$, and (iii.) as a constant infusion over the entire duration of the study. An improvement in postmeal glucose tolerance was apparent only with early administration of insulin (Fig. 3). Early insulin augmentation initially resulted in increased plasma insulin concentrations but subsequent insulin and C-peptide concentrations were lower than in the control studies.

These results have been replicated in our laboratory comparing the effect of similar doses $(0.075 \mathrm{U} / \mathrm{kg}$ lean body mass) of regular insulin and insulin analogue lispro given subcutaneously to Type 2 diabetic patients before a $50 \mathrm{~g}$ OGTT [29]. The fast-acting insulin analogue lispro provides a therapeutic tool for assessing the metabolic outcome of restoration of an early rise in plasma insulin concentrations after an oral glucose load. In spite of comparable incremental areas under the curve (AUCs), the time-course of plasma insulin concentration was much different. After injection of regular insulin, plasma insulin peaked at $120 \mathrm{~min}$, while with lispro the peak occurred at $60 \mathrm{~min}$. Plasma 

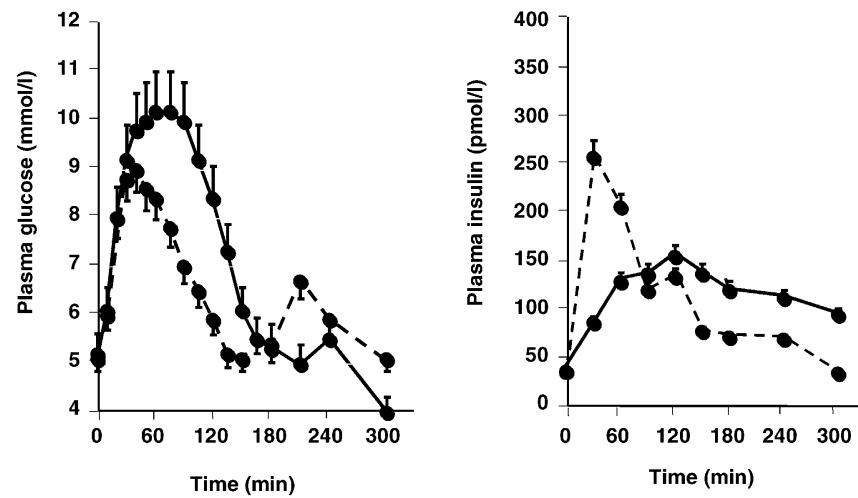

Fig. 3. Effect of superimposition of both normal and 'diabetic' post-OGTT plasma insulin profile on glucose tolerance of healthy subjects with normal glucose input. In healthy subjects, glucose was infused at a rate to mimic the rate of glucose appearance in the systemic circulation after an oral glucose load. The left hand panel illustrates the plasma glucose concentration attained when a normal (dotted line) or blunted early response (full line) of insulin is reconstructed upon suppression of endogenous insulin secretion (right hand panel). Adapted from Basu et al. [15]

insulin concentration during the last $3 \mathrm{~h}$ of the study was lower with lispro compared with regular insulin. The incremental AUC of plasma C-peptide was also lower with lispro $(p<0.01)$. The plasma glucose incremental area under the curve was $46 \%$ lower with lispro (715 \pm 109 vs $389 \pm 109 \mathrm{pmol} / 300 \mathrm{~min} ; p<0.01)$ [29] (Fig. 4). The study was carried out in combination with a double tracer technique to calculate the rates of glucose utilization and production. While there was no difference in the two studies in the overall rate of glucose disposal, the rate of EGP was suppressed in a prompter and more profound manner when lispro was administered $(p<0.05)$, particularly during the initial postprandial phase. The restoration of a prompt rise in plasma insulin concentrations was associated with an increase in glucose oxidation that was opposed to a reduction in lipid oxidation. The finding is of relevance in the light of the characteristic impairment of glucose oxidation in Type 2 diabetic patients. The enhancement of glucose oxidation was associated with a more pronounced suppression of lipid oxidation, in spite of similar reduction in plasma NEFA, suggesting that the improvement in glucose oxidation was a direct effect.

Over the past two decades, sulphonylureas have been used to enhance insulin secretion in patients with Type 2 diabetes. Data suggest that some second-generation sulphonylureas could exert a stimulatory effect on first-phase insulin secretion. A study [30] showed that first-phase insulin secretion in response to different degrees of hyperglycaemia was enhanced though not normalized by gliclazide. In our experience [31], the acute administration of gliclazide in newly diagnosed patients before receiving a constant infusion of glucose was associated with an improvement in glucose tolerance, expressed as the incremental plasma

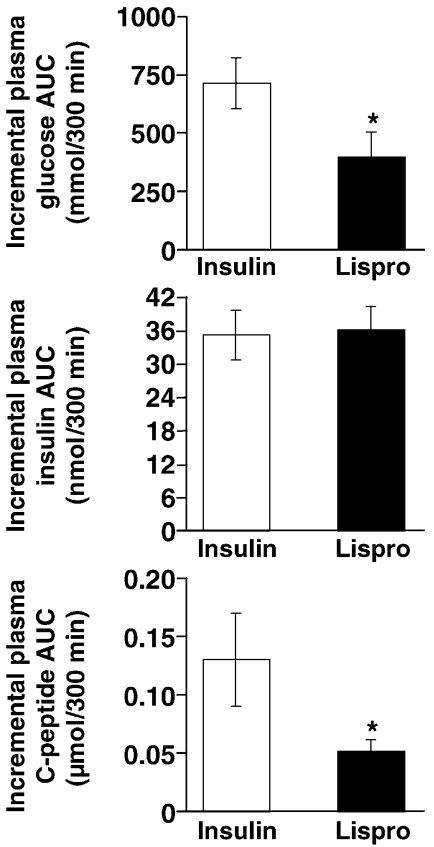

Fig. 4. Area under the incremental curve (AUC) of plasma glucose (upper panel), insulin (middle panel), and C-peptide (lower panel) after a 50-g oral glucose load preceded by subcutaneous injection of an equivalent dose $(0.075 \mathrm{U} / \mathrm{kg}$ lean body mass) of regular (open bars) and lispro (closed bars) insulin. $* p<0.05$. Reprinted with permission from Bruttomesso D et al. [29]

glucose area above baseline compared with placebo $(354 \pm 42$ vs $461 \pm 52 \mathrm{mmom} / 240 \mathrm{~min})$. However, no differences were observed in the insulin secretion rate, which only became apparent after 2 months of treatment. These data suggest that the amelioration of firstphase and second-phase insulin secretion that occurs with sulphonylureas could be partly mediated by relief of glucose toxicity.

More recently, meglitinide, a nonsulphonylurea benzoic acid derivative, has been shown to elicit an acute insulin release [32]. This compound and its analogues increase insulin secretion in a glucose-dependent manner by reducing membrane conductance in pancreatic beta cells [33]. These features suggest that meglitinide analogues could be the agents of choice for acute stimulation of insulin secretion in response to a meal. Repaglinide has been the first of these analogues made available. In an early comparative study with glibenclamide, repaglinide had a greater effect on postmeal plasma glucose concentration [34], although a more recent study reported no significant differences after one year of treatment [35]. Nateglinide, a D-phenylalanine derivative, is characterized by a rapid and short-lasting glucose dependent stimulatory effect on insulin secretion [36]. The therapeutic impact of this compound has been recently explored [37, 38]. As compared to glibenclamide, nateglinide had a greater effect on mealtime glucose excursions, though glibenclamide was associated with a greater reduction of 
fasting plasma glucose concentrations [38]. In Type 2 diabetic patients, nateglinide appeared to selectively increase early insulin release which resulted in a lower overall insulin exposure relative to glibenclamide. A direct consequence of the rapid-onset or short-duration of this insulinotropic agent was a reduction of the incidence of events suggestive of hypoglycaemia, and daytime glucose variability.

\section{Conclusions}

The early insulin release after ingestion of nutrients seems to play a critical role in the maintenance of glucose homeostasis. A prompt release of insulin is necessary to restrain postprandial glucose excursions, through a prompt inhibition of EGP, a physiological glucose disposition and, finally, in reducing a more sustained secretory stress on the beta cell. The loss of this early insulin release is a feature of the pre-diabetic condition, as it seems common in subjects with impaired glucose metabolism. These results, together with prospective findings showing that impaired early insulin release predicts diabetes, strongly indicate that the loss of this specific beta-cell function plays a critical role in decreasing glucose tolerance. This could become even more apparent if one considers that postprandial hyperglycaemia is the initial alteration in about $60 \%$ of newly diagnosed Type 2 diabetic patients [39]. The restoration of an early rise in plasma insulin concentrations is associated with improvement in glucose tolerance due to a prompter, though shortlived, suppression of EGP. This amelioration in the plasma glucose profile could prevent late hyperglycaemia and hyperinsulinaemia. Thus, therapeutic approaches aimed at restoring a physiological pattern of insulin secretion could prove effective in reducing postprandial glucose excursions in the early stage of Type 2 diabetes and possibly even prove useful in preventing diabetes.

\section{References}

1. Eriksson J, Franssila-Kallunki A, Ekstrand A et al. (1989) Early metabolic defects in persons at increased risk for non-insulin-dependent diabetes mellitus. N Engl J Med 321:337-343

2. Gulli G, Ferrannini E, Stern M, Haffner S, DeFronzo RA (1992) The metabolic profile of NIDDM is fully established in glucose-tolerant offspring of two Mexican-American NIDDM parents. Diabetes 41:1575-1586

3. Martin BC, Warram JH, Krolewski AS, Bergman RN, Soeldner JS, Kahn CR (1992) Role of glucose and insulin resistance in development of type 2 diabetes mellitus: results of a 25-year follow-up study. Lancet 340:925-929

4. DeFronzo RA, Bonadonna RC, Ferrannini E (1992) Pathogenesis of NIDDM. A balanced overview. Diabetes Care $15: 318-368$
5. Grodsky GM (1989) A new phase of insulin secretion. How will it contribute to our understanding of beta-cell function? Diabetes 38:673-678

6. Creutzfeldt W, Ebert R (1986) The enteroinsular axis. In: Van Liang W (ed) The exocrine pancreas: biology, pathobiology, and diseases. Raven Press, New York, pp 333-346

7. Bruce DG, Storlien LH, Furler SM, Chisholm DJ (1987) Cephalic phase metabolic responses in normal weight adults. Metabolism 36:721-725

8. Mitrakou A, Kelley D, Mokan M et al. (1992) Role of reduced suppression of glucose production and diminished early insulin release in impaired glucose tolerance. N Engl J Med 326:22-29

9. Del Prato S, Marchetti P, Bonadonna RC (2002) Phasic insulin release and metabolic regulation in type 2 diabetes. Diabetes [Suppl 1]:S109-S116

10. Steiner KE, Mouton SM, Bowles CR, Williams PE, Cherrington AD (1982) The relative importance of firstand second-phase insulin secretion in countering the action of glucagon on glucose turnover in the conscious dog. Diabetes 31:964-972

11. Steiner KE, Mouton SM, Williams PE, Lacy WW, Cherrington AD (1986) Relative importance of first- and second-phase insulin secretion in glucose homeostasis in conscious dog. II. Effects on gluconeogenesis. Diabetes 35:776-784

12. Consoli A, Nurjhan N, Capani F, Gerich J (1989) Predominant role of gluconeogenesis in increased hepatic glucose production in NIDDM. Diabetes 38:550-557

13. Luzi L, DeFronzo RA (1989) Effect of loss of first-phase insulin secretion on hepatic glucose production and tissue glucose disposal in humans. Am J Physiol 257:E241-E246

14. Calles-Escandon J, Robbins DC (1987) Loss of early phase of insulin release in humans impairs gucose tolerance and blunts thermic effect of glucose. Diabetes 36:1167-1172

15. Basu A, Alzaid A, Dinneen S, Caumo A, Cobelli C, Rizza RA (1986) Effects of a change in the pattern of insulin delivery on carbohydrate tolerance in diabetic and nondiabetic humans in the presence of differing degrees of insulin resistance. J Clin Invest 97:2351-2361

16. Taylor R, Magnusson I, Rothman DL et al. (1996) Direct assessment of liver glycogen storage by $13 \mathrm{C}$ nuclear magnetic resonance spectroscopy and regulation of glucose homeostasis after a mixed meal in normal subjects. J Clin Invest 97:126-132

17. DeFronzo RA, Bonadonna RC, Ferrannini E (1992) Pathogenesis of NIDDM. A balanced overview. Diabetes Care 15:318-368

18. Matthai S, Stmvoll M, Kellerer M, Haring H-U (2000) Pathophysiology and pharmacological treatment of insulin resistance. Endocr Rev 21:585-618

19. Lillioja S, Mott DM, Howard BV et al. (1988) Impaired glucose tolerance as a disorder of insulin action. Longitudinal and cross-sectional studies in Pima Indians. N Engl J Med 318:1217-1225

20. Rossetti L, Giaccari A, DeFronzo RA (1990) Glucose toxicity. Diabetes Care 13:610-630

21. Cerasi E, Luft R (1967) "What is inherited - What is added" Hypothesis for the pathogenesis of diabetes mellitus. Diabetes 16:615-627

22. Efendic S, Luft R, Wajngot A (1984) Aspects of the pathogenesis of type 2 diabetes. Endocr Rev 5:395-410

23. Lundgren $\mathrm{H}$, Bengtsson $\mathrm{C}$, Blohmè $\mathrm{G}$, Lapidus L, Waldeström J (1990) Fasting serum insulin concentration and early insulin response as risk determinants for developing diabetes. Diabet Med 7:407-413 
24. Kosaka K, Kuzuya T, Hagura R, Yoshinaga H (1996) Insulin response to oral glucose load is consistently decreased in established non-insulin-dependent diabetes mellitus: the usefulness of decreased early insulin response as a predictor of non-insulin-dependent diabetes mellitus. Diabet Med 13:S109-S119

25. Cretti A, Lehtovirta M, Bonora E et al. (2001) Assessment of beta-cell function during the oral glucose tolerance test by a minimal model of insulin secretion. Eur J Clin Invest 31:405-416

26. Bonadonna RC, Cretti A, Brunato B, Cirillo R, Del Prato S (2000) Metabolic features of impaired glucose regulation in Caucasians. Diabetologia 43 [Suppl 1]:A16 (Abstract)

27. Weyer C, Bogardus C, Mott DM, Pratley RE (1999) The natural history of insulin secretory dysfunction and insulin resistance in the pathogenesis of type 2 diabetes mellitus. J Clin Invest 104:787-794

28. Bruce DG, Chisholm DJ, Storlien LH, Kraegen EW (1988) Physiological importance of deficiency in early prandial insulin secretion in non-insulin-dependent diabetes. Diabetes 37:736-744

29. Bruttomesso D, Pianta A, Mari A et al. (1999) Restoration of early rise in plasma insulin levels improves the glucose tolerance of type 2 diabetic patients. Diabetes 48:99105

30. Hosker JP, Rudenski AS, Burnett MA, Matthews DR, Turner RC (1989) Similar reduction of first- and secondphase B-cell responses at three different glucose levels in type II diabetes and the effect of gliclazide therapy. Metabolism 38:767-772

31. Tos V da, Maran A, Vigili de Kreutzenberg S, et al. (2000) Mechanisms of acute and chronic hypoglycemic action of gliclazide. Acta Diabetol 37:201-206
32. Malaisse WJ (1995) Stimulation of insulin release by nonsulfonylurea hypoglycemic agents: the meglitinide family. Horm Metab Res 27:263-266

33. Bakkali-Nadi A, Malaisse-Lagae F, Malaisse WJ (1994) Insulinotropic action of meglitinide analogs: concentrationresponse relationship and nutrient dependency. Diabetes Res 27:81-87

34. Wolffenbuttel BH, Nijst L, Sels JP, Menheere PP, Muller PG, Kruseman AC (1993) Effects of a new oral hypoglycaemic agent, repaglinide, on metabolic control in sulphonylurea-treated patients with NIDDM. Eur J Clin Pharmacol 45:113-116

35. Wolffenbuttel BHR, Landgraf R, on behalf of the Dutch and German Repaglinide Study Group (1999) A 1-year multicenter randomized double-blind comparison of repaglinide and glyburide for the treatment of type 2 diabetes. Diabetes Care 22:463-467

36. Sato Y, Nishikawa M, Shinkai H, Sukegawa E (1991) Possibility of ideal blood glucose control by a new oral hypoglycemic agent, N-[(trans-4-isopropylcyclohexyl)-carbonyl]-Dphenylalanine (A-4166), and its stimulatory effect on insulin secretion in animals. Diabetes Res Clin Pract 12:53-59

37. Hanefeld M, Bouter KP, Dickinson S, Guitard C (2000) Rapid and short-acting mealtime insulin secretion with nateglinide controls both prandial and mean glycaemia. Diabetes Care 23:202-207

38. Hollander PA, Schwartz SL, Gatlin MR et al. (2001) Importance of early insulin secretion: comparison of nateglinide and glyburide in previously diet-treated patients with type 2 diabetes. Diabetes Care (2001) 24:983-988

39. Baron AD (1998) Postprandial hyperglycaemia and alphaglucosidase inhibitors. Diabetes Res Clin Pract 40 [Suppl]:S51-S55 